\title{
Satellite Image Compression Based On SPIHT Algorithm
}

\author{
C. Periyasamy \\ Department of Mathematics, AMET University, Chennai, India
}

\begin{abstract}
In the domains that deal with a large scale image applications force the use of image compression in order to reduce the required storage. Due to the constrained bandwidth and storage capacity in the field of marine a new compression technique for the compression of the satellite images is proposed here. In our system, we are separating the region of Region of Interest (ROI) part from the original image. And this ROI images can be used for compression scheme by an algorithm known as the Set Partitioning in Hierarchical Trees (SPIHT) algorithm. The SPIHT algorithm is a part of the Lossless compression algorithm. The performance of our method can be done by calculating the PSNR values of the output images.
\end{abstract}

Keywords: Compression, ROI, SPIHT and Lossless.

\section{Introduction}

Imaging domains that deal with the use of image compression consists of the best existing compression method, with respect to image quality and are the lossless compression methods and is discussed here. In the domains that deal with a large scale image applications force the use of image compression in order to reduce the required storage.

\subsection{Background}

The paper discussed in [1] uses the hybrid algorithm for the image compression technique. The hybrid algorithm is DWT for achieving a higher compression ratio and the DCT with the Huffman algorithm is used to preserve the quality of the reconstructed image.

A method to increase the compression ratio with less computational burden is discussed in [2]. In order to decomposition of a sub-block into equal sized bands, the DCT is used as a band pass filter and a high similarity property is found among the bands. An adaptive sampling algorithm is used in [3], for evaluating the area difference between the predicted and the correct points to decide the significant coefficients. A linear equation is employed on the decoder side for the reconstruct of the coefficients between two sequent significant coefficients. Region of interest (ROI) based on compression of image is discussed in [4] for classification applications. The method is a fusion of both lossy and lossless compression along with wavelets transforms like Embedded Zero-Tree Wavelet (EZW) is as derived techniques. An image compression method based on Integer Wavelet Transform (IWT) and SVD is discussed in [5]. A graph based quantization is used in the method but the adaptive Huffman coding is used for entropy encoding. Also the IWT used here because it is reversible. An improved SPIRT algorithm in which most of the energy is intense in the low frequency sub-band after wavelet transform is discussed in [6]. By using the methods in wavelet tree construction and the diversity judgment rules of changing the original algorithm, the initial stage of the zero tree scan number is reduced. In paper discussed in [7] uses Single Input Fuzzy Logic Controller Based SVC for Dynamic Performance Enhancement of Power Systems. An efficient video compression technique as modified HEVC coding based on saliency features to counter the existing drawbacks is introduced in [8]. Comparative Study of Wavelet Image Compression: JPEG2000 Standart using DWT and lead to an image data compression while preserving the essential quality of the original image is discussed in [9]. Discrete Wavelet Transform Based Image Compression using Frequency Band Suppression and Throughput Enhancement is described in [10]. The Security of Arithmetic Compression Based Text Steganography Method is explained in [11]. 


\subsection{The Problem}

The main drawback is that users cannot control the image quality over the background region or between regions with different degree of interest. For solving network bandwidth, security problems and storage problems, image need to be compressed.

\section{Proposed Solution}

The methodology of our proposed system is based on the lossless image compression algorithm in which the separation of the ROI regions is done from the original satellite images and is followed by the method known as SPIHT algorithm. The framework of the system is as shown in Figure 1.

\subsection{ROI Extraction}

In our proposed system the $\mathrm{ROI}$ extracted region is used for the compression process so as to increase our compression accuracy. The system is said to be as the lossless compression method. The reason for this is due to the usage of the ROI image for the compression process. From the ROI images the lossless compression is done by using the SPIHT algorithm.

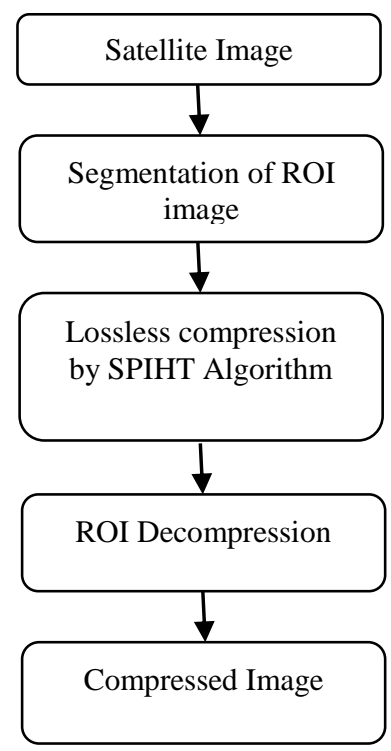

Figure 1. Block diagram for the proposed image compression Method

\subsection{SPIHT Based Compression Process}

After the extraction of the $\mathrm{ROI}$ regions, the $\mathrm{ROI}$ images are taken as the input for the SPIHT algorithm where the compression occurs. In this algorithm a progressive coding is given for processing the image corresponding to a lowering threshold. The only difference is that in the concept of zero trees at the highest level of a coefficient in a specific sub band that considers the insignificant against a specific threshold. So the pixels only with higher values are compressed without any loss of data in it. The results are as shown below for the proposed system.

\section{Results and Discussion}

The image compression technique based on the ROI image based SPIHT algorithm is said to be as the lossless compression technique in which the compression occurs without any data loss in it so that only the images are compressed without loss of data. The output result is as shown in Figure 2. 


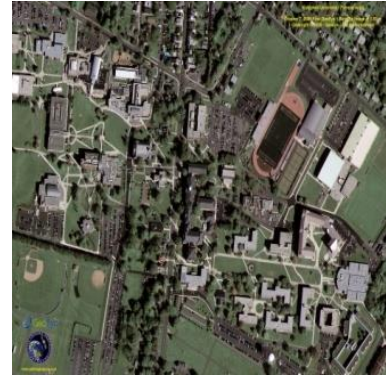

(a)

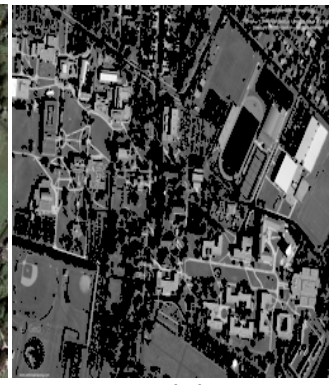

(b)

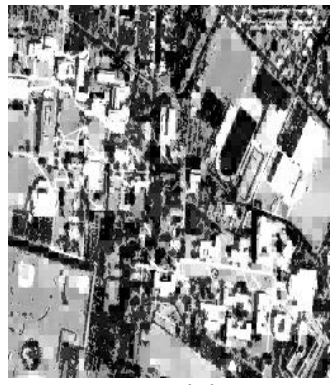

(c)

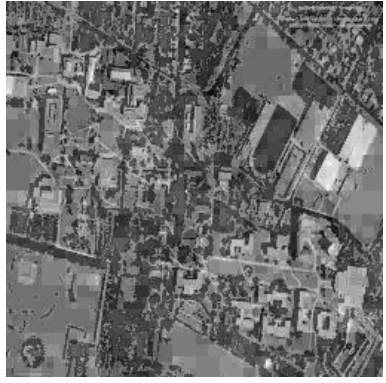

(d)

Figure 2 (a) Original image (b) Extracted ROI (c) SPIHT algorithm image (d) Compressed output image

\section{Conclusion}

The proposed image compression method based on the ROI extracted based lossless compression method by using the SPIHT algorithm is discussed. The method is tested by the literally available satellite images. And the performance of the proposed system is analyzed by calculating the PSNR values of the compressed image. Our proposed system has obtained PSNR values of about $88.6 \% \mathrm{db}$.

\section{References}

[1] Sharma S, Bhat U. Image Compression using an efficient hybrid algorithm, 2013.

[2] Wu Y G, Tai S C. Medical image compression by discrete cosine transforms spectral similarity strategy. IEEE Transactions on Information Technology in Biomedicine, 2001; 5(3): 236-243.

[3] Wu Y G. Medical image compression by sampling DCT coefficients. IEEE Transactions on Information Technology in Biomedicine, 2002; 6(1): 86-94.

[4] Reddy B V, Reddy P B, Kumar P S, Reddy A S. Lossless Compression of Medical Images for Better Diagnosis. IEEE 6th International Conference on Advanced Computing (IACC), 2016; 404-408.

[5] Savitri P A I, Murdiansyah D T, Astuti W. Digital medical image compression algorithm using adaptive Huffman coding and graph based quantization based on IWT-SVD. IEEE 4th International Conference on Information and Communication Technology, 2016; 1-5.

[6] Bin L, Qinggang M. An improved SPIHT wavelet transform in the underwater acoustic image compression. IEEE International Conference on Measurement, Information and Control (ICMIC), 2013; 2:1315-1318.

[7] Subramanian D D P. Design of a Single Input Fuzzy Logic Controller Based SVC for Dynamic Performance Enhancement of Power Systems, 2014.

[8] Rekha B. High Definition Video Compression Using Saliency Features. Indonesian Journal of Electrical Engineering and Computer Science, 2017; 7(3).

[9] Ennaciri A, Erritali M, Mabrouki M, Bengourram J. Comparative Study of Wavelet Image Compression: JPEG2000 Standart. Indonesian Journal of Electrical Engineering and Computer Science, 2015; 16(1): 83-90.

[10] Lakshmi Narayanan K, Ramesh G P. Discrete Wavelet Transform Based Image Compression using Frequency Band Suppression and Throughput Enhancement. International Journal of MC Square Scientific Research, 2017; 9(2): 176-182.

[11] Saniei R, Faez K. The Security of Arithmetic Compression Based Text Steganography Method. International Journal of Electrical and Computer Engineering, 2013; 3(6): 797. 